\title{
Atitudes de enfermeiros na administração de quimioterápicos em oncologia pediátrica
}

\author{
Nurses' attitudes in the administration of chemotherapy in pediatric oncology \\ Actitudes de enfermeros en la administración de quimioterápicos en oncología pediátrica
}

\author{
Fernanda Machado Silva-Rodrigues'; Jennifer Kamila da Silva"; Michelle Darezzo Rodrigues Nunes"II; \\ Luciana Gonzaga dos Santos Cardoso ${ }^{\prime V}$; Lucila Castanheira Nascimentov
}

\begin{abstract}
RESUMO
Objetivo: analisar as atitudes de enfermeiros acerca da administração de antineoplásicos em oncologia pediátrica. Método: estudo descritivo, com análise estatística dos dados. Participaram 20 enfermeiros de um hospital de ensino, localizado na capital paulista, que administravam quimioterapia (QT) a crianças e adolescentes. Em 2016, mediante questionário autoaplicável, foram coletados dados de caracterização dos participantes e sobre suas atividades profissionais na administração de QT, que incluíam principais receios e preocupações, além dos potenciais eventos adversos associados à quimioterapia. Resultados: poucos participantes eram especialistas na área de oncologia ou pediatria; a maioria dos profissionais buscou conhecimento e capacitação por iniciativa própria; os enfermeiros classificaram a atividade como complexa e específica para quem possui interesse na área. Conclusão: todos os enfermeiros consideraram a QT o principal tratamento para o câncer infanto-juvenil. O risco de extravasamento e a falta de dispositivos adequados para administração das drogas foram os fatores que mais preocuparam os participantes.
\end{abstract}

Descritores: Antineoplásicos; Conhecimentos, Atitudes e Prática em Saúde; Oncologia; Enfermagem pediátrica.

\section{ABSTRACT}

Objective: to examine nurses' attitudes to the administration of antineoplastics in pediatric oncology. Method: this descriptive study used statistical data analysis. The participants were twenty nurses, who administered chemotherapy to children and adolescents at a teaching hospital in São Paulo City. In 2016, data were collected using a self-report questionnaire on the participants' characteristics and their experience with chemotherapy administration, which included the main chemotherapyrelated fears, concerns and potential adverse events. Results: few participants were specialists in the field of oncology or pediatrics; most sought knowledge and capacity-building on their own initiative; and the nurses classified the activity as complex and specific to those with an interest in the field. Conclusion: all the nurses considered chemotherapy the main treatment for childhood cancer. The factors that most concerned the participants were the risk of extravasation and the lack of appropriate devices for drug administration.

Descriptors: Antineoplastic agents; health knowledge, attitudes and practice; medical oncology; pediatric nursing.

\section{RESUMEN}

Objetivo: analizar las actitudes de enfermeros acerca de la administración de antineoplásicos en oncología pediátrica. Método: estudio descriptivo, con análisis estadística de los datos. Participaron 20 enfermeros de un hospital de enseñanza, ubicado en la capital de São Paulo, que administraban quimioterapia (QT) a niños y adolescentes. En 2016, a través de cuestionario autoaplicable, se recopilaron datos sobre la caracterización de los participantes y sobre sus actividades profesionales en la administración de quimioterapia que incluían los principales temores, preocupaciones y potenciales eventos adversos asociados a la quimioterapia. Resultados: pocos participantes eran especialistas en el área de oncología o pediatría; la mayoría de los profesionales buscó conocimiento y capacitación por iniciativa propia; los enfermeros clasificaron la actividad como compleja y específica para quien tiene interés en el área. Conclusión: todos los enfermeros consideraron la quimioterapia como el tratamiento principal para el cáncer infantil. El riesgo de extravasación y la falta de dispositivos adecuados para la administración de las drogas fueron los factores que más preocuparon a los participantes.

Descriptores: Antineoplásicos; conocimientos, actitudes y práctica en salud; oncología médica; enfermería pediátrica.

\section{INTRODUÇÃO}

O câncer infanto-juvenil corresponde a um grupo de várias doenças que têm em comum a proliferação desordenada de células anormais ${ }^{1}$. No Brasil, é a segunda causa de morte em crianças e adolescentes, entre um e dezenove anos de idade, estando a primeira relacionada a violência e acidentes ${ }^{1,2}$. Além disso, é um acontecimento avassalador que provoca mudanças e reações inesperadas, não apenas na vida da criança, mas também na vida de seus familiares ${ }^{2}$.

'Enfermeira. Doutora em Ciências. Faculdade de Ciências Médicas da Santa Casa de São Paulo, Brasil. E-mail: fernanda.rodrigues@fcmsantacasasp.edu.br "Enfermeira. Residente. Programa de Residência Multiprofissional em Oncologia Pediátrica. Instituto de Oncologia Pediátrica. São Paulo, Brasil. E-mail: jennifer-kamila@live.com

I'Enfermeira. Doutora em Ciências. Faculdade de Enfermagem da Universidade do Estado do Rio de Janeiro, Brasil. E-mail: mid13@hotmail.com

IVEnfermeira. Mestre em Ciências. Faculdade de Ciências Médicas da Santa Casa de São Paulo. Brasil. E-mail: lucianagcardoso@uol.com.br

vEnfermeira. Doutora em Ciências. Escola de Enfermagem de Ribeirão Preto, Universidade de São Paulo. Brasil. E-mail: lucila@eerp.usp.br 
Atualmente várias modalidades de tratamento são empregadas para o tratamento do câncer infanto-juvenil, dentre as principais, a quimioterapia, a cirurgia e a radioterapia, a depender do tipo de tumor e da extensão da doença $^{3}$. No entanto, a quimioterapia é a modalidade mais utilizada, podendo ser associada ou não às outras modalidades de tratamento ${ }^{3,4}$

Apesar do uso de drogas citotóxicas aumentarem o sucesso na resposta terapêutica contra o câncer infantil, tais drogas atuam destruindo ou inibindo o crescimento de células que se multiplicam rapidamente, sem diferenciar células cancerígenas de células saudáveis, o que leva a efeitos adversos, tais como, a mielossupressão, náuseas, vômitos, diarreia e alopecia 5 .

A administração de drogas citotóxicas, principalmente a crianças pequenas, é considerada uma tarefa complexa, que requer conhecimentos específicos, além de apresentar maior potencial para erros e transgressões aos princípios de segurança do paciente pediátrico ${ }^{2,3}$. Adicionalmente, a administração de tais drogas integra um complexo processo que também compreende as precauções ocupacionais, devido aos riscos associados à exposição a esses agentes ${ }^{3}$.

Os enfermeiros são os principais responsáveis por zelar para que o procedimento da administração de quimioterápicos seja seguro a todos os envolvidos, provendo o suporte necessário e capacitando o paciente e sua família para lidar com as complicações desta modalidade de tratamento ${ }^{2}$. Diante do exposto, este estudo se propôs a analisar as atitudes de enfermeiros acerca da administração de antineoplásicos em oncologia pediátrica.

\section{REVISÃO DE LITERATURA}

Por desempenhar papel de extrema importância na administração dos quimioterápicos, é essencial que o enfermeiro possua conhecimentos e habilidades suficientes para planejar suas ações e avaliar o paciente oncológico pediátrico em sua integralidade ${ }^{1-3}$.

Estudo britânico, com enfermeiros que atuavam em oncologia pediátrica, apontou que os profissionais se sentiam ansiosos e preocupados quanto à ocorrência de erros durante a administração de quimioterápicos ${ }^{6}$. Outro estudo $^{7}$, indicou que administrar quimioterápicos era uma atividade estressante para alguns enfermeiros, contrária ao lado recompensador de cuidar desses pacientes e de suas famílias. Dentre os principais fatores causadores de estresse, foram citados: os efeitos adversos causados pelas drogas, o baixo nível de informação dos pacientes e de suas famílias e o conhecimento limitado dos próprios profissionais sobre a terapêutica?

Alguns estudos evidenciam o quanto os profissionais que prestam assistência a pacientes oncológicos pediátricos devem possuir conhecimentos dos mecanismos de ação das drogas e estarem atualizados sobre as novas tecnologias, não só para executar o procedimento de administração de quimioterápicos com perícia, mas também oferecer cuidados adequados às necessidades desta clientela, principalmente aqueles relacionados ao reconhecimento e manejo dos efeitos adversos da terapia ${ }^{4,5,7}$. Há, no entanto, poucas evidências sobre as estratégias educacionais que contribuem para a formação dos enfermeiros neste campo do saber ${ }^{7,8}$.

Os Estados Unidos da América (EUA) estão dentre os poucos países que possuem cursos para o desenvolvimento de competências para a administração de quimioterápicos a pacientes pediátricos ${ }^{7}$. Já os países europeus, em sua maioria, não oferecem treinamentos padronizados a seus enfermeiros, uma vez que os hospitais adotam protocolos e programas educacionais próprios ${ }^{6}$.

Não obstante o protagonismo do enfermeiro na administração da terapia antineoplásica em oncologia pediátrica, suas atitudes, seu conhecimento e a sua capacitação para realizar tal procedimento ainda se encontram insuficientemente descritos na literatura, principalmente no contexto nacional.

\section{METODOLOGIA}

Estudo descritivo, com análise estatística dos dados, realizado no Serviço de Oncologia Pediátrica de um hospital de ensino, localizado na cidade de São Paulo, em 2016. A pesquisa recebeu aprovação do Comitê de Ética em Pesquisa em Seres Humanos, sob o parecer no 1.396.366.

Dos 21 enfermeiros que administravam terapia antineoplásica como parte de suas atividades assistenciais às crianças e adolescentes com câncer, apenas um não integrou o estudo, pois encontrava-se em período de férias na ocasião da coleta dos dados.

Para a coleta de dados foi elaborado um questionário auto preenchido, cujo conteúdo baseou-se na literatura já publicada sobre a temática ${ }^{4,6} .0$ instrumento contemplou itens relativos aos dados de caracterização dos profissionais (tempo de formação, tempo de experiência na administração de antineoplásicos e nível de formação); questões a respeito da administração de tais drogas; principais receios e preocupações ao administrá-las na população pediátrica; circunstâncias que causavam insegurança ao administrá-las; sentimentos vivenciados na primeira administração de 
antineoplásicos; conhecimentos sobre eventos adversos ou toxicidades comuns nas crianças submetidas a tratamento com drogas citotóxicas e a finalidade da quimioterapia no tratamento do câncer infanto-juvenil segundo os profissionais.

Os dados foram digitados em planilha do Microsoft Office Excel (versão 2014) e submetidos à análise de consistência mediante dupla digitação. As planilhas foram comparadas para identificação e correção de possíveis divergências. Utilizou-se a estatística descritiva para a análise dos dados.

\section{RESULTADOS}

Dos 20 enfermeiros incluídos no estudo, 8 (40 \%) tinham tempo de formação maior que 10 anos, experiência na administração de drogas citotóxicas de um a cinco anos e maioria não possuía especialização na área de pediatria, nem de oncologia 11 - (55\%) conforme mostra a Tabela 1.

TABELA 1: Caracterização dos participantes. São Paulo, 2016.

\begin{tabular}{|c|c|}
\hline Variáveis & $f(\%)$ \\
\hline \multicolumn{2}{|l|}{ Sexo } \\
\hline Feminino & 19 (95) \\
\hline Masculino & $1(5)$ \\
\hline \multicolumn{2}{|l|}{ Tempo de formação } \\
\hline$>10$ anos & $8(40)$ \\
\hline 6 a 10 anos & 7 (35) \\
\hline 1 a 5 anos & $5(25)$ \\
\hline \multicolumn{2}{|c|}{ Tempo de administração de drogas citotóxicas } \\
\hline$>10$ anos & $3(15)$ \\
\hline 6 a 10 anos & $5(25)$ \\
\hline 1 a 5 anos & $8(40)$ \\
\hline$<1$ ano & $4(20)$ \\
\hline \multicolumn{2}{|l|}{ Formação (Especialização) } \\
\hline Em pediatria & 7 (35) \\
\hline Em oncologia & $2(10)$ \\
\hline Outras ${ }^{(*)}$ & $6(30)$ \\
\hline Não tinham especialização & $5(25)$ \\
\hline
\end{tabular}

Os enfermeiros foram questionados quanto aos sentimentos vivenciados durante as primeiras administrações de quimioterápicos. Medo, receio e insegurança foram às respostas mais frequentes $11(38,0 \%)$ conforme descreve a Tabela 2.

Apesar da maioria dos enfermeiros não ter formação especializada em pediatria ou oncologia, apenas 3(6,7\%), afirmaram que não estavam preparados para a primeira administração de QT. A busca de conhecimento por iniciativa própria foi a estratégia utilizada pela maioria dos profissionais $12(75,0 \%)$ para minimizar a insegurança ao executar a técnica como descreve a Tabela 2.

Para a maioria dos enfermeiros, a administração de drogas antineoplásicas foi considerada complexa e específica para quem tem interesse na área de oncologia pediátrica $16(84,2 \%)$. Porém, para 2 (10,5\%) profissionais a administração de QT é similar à administração de outras medicações em pediatria, o que pode ser visto na Tabela 3.

O risco de extravasamento foi considerado o principal receio nas primeiras administrações de quimioterápicos para 17 (37,9\%) enfermeiros, e também a principal preocupação relatada na administração de antineoplásicos para 17 $(37,9 \%)$ dos profissionais, perfazendo $45,9 \%$ dos códigos. 
TABELA 2: Sentimentos, receios e busca por auxílio nas primeiras administrações de quimioterápicos em crianças. São Paulo, 2016

\begin{tabular}{|c|c|}
\hline Variáveis & $f(\%)$ \\
\hline \multicolumn{2}{|l|}{ Sentimentos } \\
\hline Medo de possíveis reações adversas das drogas no paciente pediátrico & $6(20,7)$ \\
\hline Receio e insegurança & $5(17,3)$ \\
\hline Preocupação concernente a aspectos emocionais do paciente pediátrico e de sua família & $5(17,3)$ \\
\hline Segurança e tranquilidade & $5(17,2)$ \\
\hline Sensibilização por se tratar de criança & $3(10,3)$ \\
\hline Não se lembra ou não soube descrever & $3(10,3)$ \\
\hline Responsabilidade em relação a restaurar a saúde da criança & $2(6,9)$ \\
\hline Total de sentimentos & $29(100,0)$ \\
\hline \multicolumn{2}{|l|}{ Receios } \\
\hline Extravasamento & $17(38,6)$ \\
\hline Reações adversas na criança & $14(31,8)$ \\
\hline Desconhecimento dos quimioterápicos & $4(9.1)$ \\
\hline Receios de riscos ocupacionais (autoexposição aos antineoplásicos) & $4(9.1)$ \\
\hline Percepção de que não estava preparado para realizar a atividade & $3(6,8)$ \\
\hline Desconhecimento dos cuidados recomendados para a administração & $2(4,6)$ \\
\hline Total de receios & $44(100,0)$ \\
\hline \multicolumn{2}{|l|}{ Forma de lidar com receios } \\
\hline Busca de conhecimento por iniciativa própria & $12(75,0)$ \\
\hline Aprendizado na prática & $4(25,0)$ \\
\hline Total de forma de lidar com receios & $16(100)$ \\
\hline
\end{tabular}

Apenas 1 (2,8\%) profissional declarou sentir-se inseguro ao administrar quimioterápicos. Ao serem questionados especificamente sobre o momento em que se sentem mais inseguros para administrar drogas antineoplásicas, 8 $(44,4 \%)$ enfermeiros referiram insegurança quando o setor apresenta elevado número de pacientes conforme a Tabela 3.

Considerando a experiência de cada participante, questionou-se o que pensavam a respeito dos quimioterápicos como terapêutica para o câncer infanto-juvenil, e os 20 (100\%) participantes mencionaram que a quimioterapia é o principal tratamento para a maioria das neoplasias na infância.

Os efeitos adversos foram o segundo principal receio dos profissionais, citado por 14 (70\%) enfermeiros, compondo $31,3 \%$ dos códigos encontrados. Especificamente em relação aos eventos adversos vivenciados na administração de antineoplásicos, as alterações gastrointestinais, como náuseas e vômitos, foram referidas por todos os profissionais 20 (100\%), compondo $37 \%$ dos códigos identificados, seguidas de hipersensibilidade e anafilaxia citada por 8 (40\%) dos enfermeiros (14,8\% dos códigos) e extravasamento ou infiltração de drogas vesicantes por 5 enfermeiros (8,6\% dos códigos) de acordo com Tabela 3.

\section{DISCUSSÃO}

O presente estudo buscou descrever as atitudes de enfermeiros em relação à administração de drogas antineoplásicas em oncologia pediátrica. A literatura sobre o tema, tanto em termos de manuais e diretrizes específicas, quanto de artigos científicos, ainda é bastante incipiente, principalmente na língua portuguesa.

As características sociodemográficas da amostra investigada foram: profissionais de enfermagem de ambos os sexos, adultos, com tempo de formação entre um ano e mais de dez anos. Predominaram profissionais do sexo feminino, característica histórica no exercício da enfermagem e que persiste nos dias atuais ${ }^{8}$.

Embora o tempo de formação dos enfermeiros tenha variado de um ano a mais de 10 anos, nenhum dos participantes trabalhou exclusivamente no cuidado a pacientes oncológicos pediátricos. Ainda que a maior parte tivesse o título de especialista, observou-se que nenhum dos enfermeiros se especializou na área de oncologia pediátrica, algo que se justifica pela pequena oferta de cursos de pós-graduação lato sensu nesta área, geralmente oferecidos nas grandes capitais.

Apenas dois profissionais eram especialistas em oncologia, formação que potencialmente melhor os capacita para a administração de quimioterápicos, o que diverge do apontado em estudo realizado no Reino Unido, em que a maioria (64\%) dos enfermeiros participantes da pesquisa era especialista nessa área ${ }^{6}$. Neste mesmo estudo, em se 
tratando da administração de drogas citotóxicas, os enfermeiros apresentaram, sobretudo, preocupações concernentes a aspectos emocionais do paciente pediátrico e de sua família ${ }^{6}$, o que vai de encontro com os achados desta pesquisa, em que se observou que os profissionais com especialização em pediatria ou oncologia demonstraram maior preocupação com os aspectos emocionais de seus pacientes e familiares.

TABELA 3. Classificação da atividade, preocupações, insegurança e efeitos adversos vivenciados na administração de quimioterápicos. São Paulo, 2016.

\begin{tabular}{|c|c|}
\hline Variáveis & $f(\%)$ \\
\hline \multicolumn{2}{|l|}{ Classificação da atividade } \\
\hline Específica para quem tem interesse na área de oncologia pediátrica & $9(47,4)$ \\
\hline Complexa & $7(36,8)$ \\
\hline Similar à administração de outras medicações em pediatria & $2(10,5)$ \\
\hline Desafiadora & $1(5,3)$ \\
\hline Total de classificação da atividade & $19(100)$ \\
\hline \multicolumn{2}{|l|}{ Preocupações } \\
\hline Riscos de extravasamento das drogas envolvidas & $17(45,9)$ \\
\hline Falta de dispositivos e acessos venosos apropriados & $10(27,0)$ \\
\hline Desconhecer os cuidados e as particularidades das drogas administradas & $6(16,2)$ \\
\hline Desconhecer os possíveis eventos adversos das drogas & $3(8,1)$ \\
\hline Insegurança ao administrar quimioterápicos & $1(2,8)$ \\
\hline Total de preocupações & $37(100)$ \\
\hline \multicolumn{2}{|l|}{ Insegurança } \\
\hline Quando o setor apresenta elevado número de pacientes & $8(44,4)$ \\
\hline Quando a escala de pessoal está reduzida & $6(33,3)$ \\
\hline Durante os plantões noturnos & $2(11,1)$ \\
\hline Não me sinto inseguro ao administrar quimioterapia & $2(11,1)$ \\
\hline Total de insegurança & $18(100)$ \\
\hline \multicolumn{2}{|l|}{ Efeitos Adversos } \\
\hline Alterações gastrointestinais & $20(39,2)$ \\
\hline Hipersensibilidade ou anafilaxia & $8(15,7)$ \\
\hline Extravasamento ou infiltração de droga vesicante & $5(9,8)$ \\
\hline Alterações hematológicas & $3(5,9)$ \\
\hline Alterações metabólicas & $3(5,9)$ \\
\hline Indisposição & $2(3,9)$ \\
\hline Anorexia & $2(3,9)$ \\
\hline Febre & $2(3,9)$ \\
\hline Alterações emocionais & $2(3,9)$ \\
\hline Eventos nunca ou raramente associados à terapia com antineoplásicos & $2(3,9)$ \\
\hline Alopecia & $1(2,0)$ \\
\hline Hipotensão & $1(2,0)$ \\
\hline Total de efeitos adversos & $51(100)$ \\
\hline
\end{tabular}

Acredita-se que, especializar-se em uma determinada área de atuação, pode favorecer a compreensão dos enfermeiros a respeito das peculiaridades inerentes ao cuidar em determinadas especialidades. Ou seja, a atenção em relação aos aspectos emocionais, tanto da criança quanto de seus familiares, mostra-se como um aspecto que transcende o saber técnico relacionado à administração dos quimioterápicos ${ }^{2,6}$.

Destacaram-se o medo dos extravasamentos e da ocorrência de reações adversas. Ao contrário de outros estudos publicados na literatura ${ }^{3,4,6}$, poucos enfermeiros demonstraram despreparo para a administração de quimioterápicos, e a maioria referiu buscar conhecimentos por iniciativa própria.

O risco de extravasamento e a falta de dispositivos e acessos venosos adequados para a administração de quimioterápicos foram alguns dos fatores mais preocupantes para os participantes. Ressalta-se que esses dois fatores merecem atenção, uma vez que a ausência de uma rede venosa preservada e de dispositivos adequados para os 
acessos venosos, aumentam os riscos de ocorrência de extravasamentos, os quais representam consequências graves ao paciente pediátrico, dentre elas, a necrose tecidual ${ }^{4,6}$.

$\mathrm{Na}$ busca por auxílio para enfrentar seus receios e inseguranças, os profissionais tentaram adquirir conhecimentos por iniciativa própria, o que expressa preocupação e um movimento para melhorar a assistência que, consequentemente, poderá reduzir as chances de erros na administração de drogas citotóxicas. Corroborando com este achado, um estudo recente sobre a educação permanente de enfermeiros oncológicos identificou que as estratégias de educação fornecidas pelas instituições hospitalares se baseiam em treinamentos admissionais, cursos e eventos científicos, sobre diversos assuntos, não havendo preparo específico em quimioterapia, o que leva os profissionais a buscarem essa competência em instituições especializadas ${ }^{9}$. Contrapondo-se ao encontrado em outros estudos internacionais, em que os enfermeiros recebiam conhecimento atualizado e regular, além da oportunidade de conviver no ambiente de trabalho com profissionais mais experientes na administração de quimioterápicos, o que sinaliza que a competência para esta atividade resulta não apenas da experiência clínica, mas também de atividades educativas e de atualização de práticas ${ }^{3,4,6}$.

É fundamental que as instituições proporcionem meios para a capacitação constante desses profissionais, além de treinamentos e orientações específicas, principalmente relacionadas ao manejo de efeitos adversos e intercorrências no tratamento antineoplásico ${ }^{10,11}$.

Ao indicarem os momentos em que se sentiam mais inseguros para administrar drogas citotóxicas, os participantes mencionaram situações nas quais o setor apresentava elevado número de pacientes ou redução da escala de pessoal. Tais achados corroboram os resultados de outro estudo, no qual os profissionais afirmaram que o grande volume de trabalho, a falta de funcionários e o elevado número de pacientes são fatores que comprometem a administração segura dos quimioterápicos ${ }^{3}$. Adicionalmente, outros estudos apontam maior insegurança dos enfermeiros na administração de quimioterapia durante os plantões noturnos ${ }^{6,7,9,10}$

Funcionários de um hospital público e geral, muitas vezes não desempenham algumas atividades assistenciais por escolha, mas por uma necessidade institucional, frente à escassez de pessoal em determinados setores, por exemplo. Por isso, é compreensível que alguns participantes tenham classificado a administração de drogas antineoplásicas como complexa e específica para quem tem especial interesse na área de oncologia pediátrica, o que demonstra que reconhecem as especificidades da administração de tais drogas a esta população.

O estudo evidenciou que a tentativa de treinar enfermeiros não especialistas e sem interesse na área de oncologia pediátrica é desafiadora, por se tratar de uma prática especialmente complexa e com grande potencial para erros $^{6}$. A administração de quimioterápicos a crianças e adolescentes, especialmente crianças pequenas, é considerada ainda mais difícil para o enfermeiro, devido as particularidades desta faixa etária e as diferentes fases do desenvolvimento infantil ${ }^{12}$. O enfermeiro deve estar seguro de suas atitudes, suas competências e habilidades técnicas durante a realização deste procedimento ${ }^{4,6}$. Inclui-se nesse processo, o uso de sua capacidade de julgamento, energia, experiência e motivação, necessárias para responder às demandas exigidas no exercício de atividades profissionais complexas ${ }^{9,13}$

A literatura ainda preconiza que as drogas citotóxicas sejam administradas exclusivamente por enfermeiros especialistas, por considerar que estes, além de possuírem o conhecimento específico sobre o tratamento antineoplásico, tem maior domínio dos aspectos relacionados a cuidar do paciente pediátrico e de sua família ${ }^{2,7}$. Outro estudo apontou que o conhecimento do enfermeiro sem especialização acerca do uso e da importância das drogas antineoplásicas pode ser limitado, o que aumenta as chances de erros e a falta de controle frente a situações adversas ${ }^{3}$.

Sendo assim, a formação especializada é essencial para os enfermeiros que atuam nessa área, a qual requer ampla compreensão dos procedimentos, além de habilidades avançadas e fundamentadas em princípios científicos ${ }^{10,13,14}$.

Todos os profissionais consideraram a quimioterapia o principal tratamento para o câncer infanto-juvenil, em concordância com outras pesquisas que ressaltaram o importante papel desempenhado pelos antineoplásicos no tratamento das doenças oncológicas pediátricas ${ }^{7,9,14,15}$.

É de extrema importância o conhecimento dos enfermeiros para o direcionamento do cuidado, pois se este profissional não for suficientemente competente para administrar os quimioterápicos, poderá comprometer a segurança do procedimento, aumentar as chances de erros de medicação e os danos ao paciente poderão ser maiores, além de aumentar as fontes geradoras de estresse para a criança e sua família ${ }^{4,6,16}$

Os participantes do estudo restringiram-se às toxicidades e aos efeitos adversos mais comuns, como os gastrointestinais (náuseas e vômitos), que não são considerados os mais graves ou que necessitam de assistência 
especializada por parte do enfermeiro ${ }^{10,13,17}$. Este resultado converge com outros estudos que abordaram as necessidades educacionais de enfermeiros que atuam em oncologia pediátrica, em países com perfis semelhantes ao do Brasil ${ }^{14-16}$. Para os participantes de dois desses estudos, a administração de QT e o conhecimento das possíveis reações adversas associadas às drogas antineoplásicas foram apontados como prioridade de treinamento e atualização ${ }^{14,16}$

Sabe-se que os efeitos adversos relacionados aos quimioterápicos, vão muito além dos gastrointestinais, dentre eles estão a fadiga, a dor, as alterações no sono, a mucosite, a perda de peso e a alopecia, todos esses, merecem destaque e atenção dos enfermeiros, uma vez que comprometem muito a qualidade de vida da do paciente pediátrico ${ }^{18,19}$.

Faz-se importante ressaltar ainda que, de acordo com a literatura, reações de hipersensibilidade e anafilaxia, citadas como relevantes pelos participantes do presente estudo, não são eventos comuns e restringem-se a apenas alguns quimioterápicos, por exemplo, a L-Asparaginase ${ }^{17}$, quimioterápico bastante empregado no tratamento das leucemias na infância.

Os principais achados dessa pesquisa vão de encontro à constante necessidade de investimentos na qualificação e atualização dos profissionais pelas instituições de saúde. A utilização de protocolos operacionais e a promoção de treinamentos e atividades de educação permanente são exemplos de estratégias essenciais para qualificar os cuidados prestados a pacientes oncológicos pediátricos ${ }^{9,20,21}$.

Sabe-se que o câncer infantil é uma doença rara, porém a complexidade e a especificidade do cuidado nesta especialidade são indiscutíveis; acresce-se a relevância da necessidade de treinamento e/ou atualização em relação à administração de quimioterápicos ${ }^{10,22}$. Não se considera o reduzido tamanho da amostra um fator limitante do presente estudo, pois mesmo em hospitais especializados no tratamento do câncer infantil, não são todos os enfermeiros que atuam diretamente na administração da terapia antineoplásica a crianças e adolescentes.

Aspectos como a segurança do profissional na administração de QT em pediatria podem ser objeto de futuras investigações, porém não fizeram parte do escopo deste estudo, cujo objetivo foi descrever, essencialmente, as atitudes de enfermeiros frente a um importante procedimento que integra as atividades assistenciais em oncologia pediátrica.

\section{CONCLUSÃO}

Este é o primeiro estudo desta natureza no Brasil e um dos poucos disponíveis na literatura. Portanto, consiste na abordagem inicial de um tema de extrema relevância para enfermeiros atuantes nesta área.

Em oncologia pediátrica, tanto os cuidados específicos envolvidos na administração de drogas citotóxicas quanto às intervenções realizadas diante da ocorrência de possíveis eventos adversos precisam estar respaldados por conhecimentos científicos. Some-se o conhecimento da terapia antineoplásica na infância ao conhecimento das peculiaridades clínicas, de crescimento e de desenvolvimento do público pediátrico.

Sobre as atitudes dos enfermeiros frente à administração de QT em oncologia pediátrica, destacaram-se os seguintes aspectos: poucos eram especialistas na área de oncologia ou pediátrica; a busca de conhecimento por iniciativa própria foi a estratégia adotada pela maioria para melhor capacitação; os enfermeiros classificaram o procedimento como complexo e específico para quem tem especial interesse na área de oncologia pediátrica; o risco de extravasamento e a falta de dispositivos adequados para administração das drogas foram os fatores que mais preocuparam os participantes e todos os enfermeiros consideraram a quimioterapia o principal tratamento para 0 câncer infanto-juvenil.

Conclui-se, diante da complexidade e especificidade desta atividade, que é indispensável o investimento na capacitação profissional, além da realização de pesquisas futuras que abordem as necessidades educativas de enfermeiros quanto à administração de quimioterápicos a esses pacientes.

\section{REFERÊNCIAS}

1. INCA. Protocolo de diagnóstico precoce do câncer pediátrico. Brasília, DF, Brasil. [Internet]. 2017 [cited 2019 Oct 04]. Available from: https://www.inca.gov.br/publicacoes/livros/protocolo-de-diagnostico-precoce-do-cancer-pediatrico

2. Cruz EF, Silva LF, Goes FGB, Aguiar RSB, Moraes JRMM. Nursing orientation given to children in antineoplastic chemotherapy treatment. Rev. Eletr. Enf. [Internet]. 2014 [cited 2019 Sep 15]; 16(2):378-85. Available from: http://dx.doi.org/10.5216/ree.v16i2.27009. DOI: http://dx.doi.org/10.5216/ree.v16i2.27009

3. Belderson K, Billett, AL. Chemotherapy safety standards: a pediatric perspective. J. Pediatr. Oncol. Nurs. [Internet]. 2017 [cited 2019 Sep 15]; 34(3):156-9. DOI: https://dx.doi.org/10.1177/1043454217697670 
4. Kapucu S, Özkaraman AÖ, Uysal N, Bagcivan G, Şeref FÇ, Elöz A. Knowledge level on administration of chemotherapy through peripheral and central venous catheter among oncology nurses. Asia Pac. J. Oncol. Nurs. [Internet]. 2017 [cited 2019 Oct 05]; 4(1):61-8. DOI: http://dx.doi.org/10.4103/2347-5625.199081

5. Samantarath P, Pongthavornkamol K, Olson K, Sriyuktasuth A, Sanpakit K. Multiple symptoms and their influences on healthrelated quality of life in adolescents with hematologic malignancies undergoing chemotherapy. Pac. Rim. Intern. J. Nurs. Res. [Internet]. 2018 [cited 2019 Oct 05]; 22(4): 319-31. Available from: https://www.tcithaijo.org/index.php/PRIJNR/article/view/88211/109033

6. Gibson F, Shipway L, Aldiss S, Hawkins J, King W, Parr M, et al. Exploring the work of nurses who administer chemotherapy to children and young people. Eur. J. Oncol.Nurs. [Internet]. 2013 [cited 2018 Ago 20]; 17(1):59-69. DOI: https://doi.org/10.1016/j.ejon.2012.01.006

7. Andam R, Silva M. A journey to pediatric chemotherapy competence. J. Pediatr. Nurs. [Internet]. 2008 [cited 2018 Ago 15]; 23(4):257-68. DOI: https://doi.org/10.1016/j.pedn.2006.12.005

8. Fundação Oswaldo Cruz. Perfil da Enfermagem no Brasil [Internet]. 2016 [cited 2018 Jun 12]. Available from: http://portal.fiocruz.br/pt-br/content/pesquisa-inedita-traca-perfil-da-enfermagem-no-brasil

9. Santos, FC; Laus, AM; Bernardes, A; Camelo, SHH. Educación Continua: construcción del conocimiento y estrategias educativas para las enfermeras de oncología. Cultura De Los Cuidados [Internet]. 2018 [cited 2019 Oct 02]; (51): 22-30. Available from: http://ciberindex.com/c/cc/51022cc

10. Souza NR, Bushatsky M, Figueiredo EG, Melo JT, Freire DA, Santos ICRV. Oncological emergency: the work of nurses in the extravasation of antineoplastic chemotherapeutic drugs. Esc. Anna Nery Rev. Enferm. [Internet]. 2017 [cited 2018 Jun 10]; 21(1): e20170009. Available form: http://www.scielo.br/pdf/ean/v21n1/1414-8145-ean-21-01-e20170009.pdf

11. Jomar RT, Santos LS, Conteiro MF, Matsumoto KS, Gallasch CH, Taets GGC. Intraoperative hyperthermic intraperitoneal chemotherapy: what nurses should know. Rev. enferm. UERJ. [Internet]. 2017 [cited 2019 Sep 12]; 25: e29326. DOI: http://dx.doi.org/10.12957/reuerj.2017.29326

12. Boyle DA, Bush NJ. Reflections on the emotional hazards of pediatric oncology nursing: Four decades of perspectives and potential. J. Pediatr. Nurs. [Internet]. 2018 [cited 2018 Jun 15]; 40:63-73. DOI: https://doi.org/10.1016/j.pedn.2018.03.007

13. McCulloch R, Hemsley J, Kelly P. Symptom management during chemotherapy. J. Paediatr. Child Health. [Internet]. 2014 [cited 2018 Jul 13]; 24(4):166-71. DOI: https://doi.org/10.1016/j.paed.2013.10.007

14. Challinor JM, Hollis R, Freidank C, Verhoeven C. Educational needs and strategies of pediatric oncology nurses in low-and middle-income countries. An International Society of Pediatric Oncology- Pediatric Oncology in Developing Countries Nursing Working Group Initiative. Cancer Nurs. [Internet]. 2014 [cited 2018 Ago 10]; 374:36-47. DOI: https://doi.org/10.1097/NCC.0000000000000100

15. Day SW, Garcia J, Antillon F, Wilimas JA, McKeon LM, Carty RM et al. A sustainable model for pediatric oncology nursing education in low-income countries. Pediatr. Blood \& Cancer. [Internet]. 2012 [cited 2018 Ago 15]; 58(2):163-6. DOI: https://doi.org/10.1002/pbc.24007

16. Day S, Challinor J, Hollis R, Abramovitz L, Hanaratri Y, Punjwani R. Paediatric oncology nursing care in low-and middle-income countries: a need for baseline standards.Cancer Control. [Internet]. 2015 [cited 2018 Ago 15]; 111-116. Available from: http://www.cancercontrol.info/wp-content/uploads/2015/07/111-116-Day_cc2015.pdf

17. Shinnick SE, Browning ML, Koontz SE. Managing hypersensitivity to Asparaginase in pediatrics, adolescents, and young adults. J.Pediatr. Oncol.Nurs. [Internet]. 2013 [cited 2018 Ago 3]; 30(2):63-77. DOI: https://doi.org/10.1177/1043454212471728

18. Torres V, Nunes MDR, Silva-Rodrigues FM, Bravo L, Adlard K, Secola, R et al. (2019) Frequency, Severity, and Distress Associated with Physical and Psychosocial Symptoms at Home in Children and Adolescents with Cancer. Journal of Pediatric Health Care [Internet]. 2019 [cited 2019 Oct 02]; 33(4):404-14. DOI: https://doi.org/10.1016/j.pedhc.2018.11.007

19. Nunes MDR, Jacob E, Bomfim EO, Lopes-Junior LC, Lima LAG, Floria-Santos M, et al. Pain, Sleep Patterns, and HRQOL In Pediatric Patients With Cancer. European Journal of Cancer Care [Internet]. 2019 [cited 2019 Oct 02]; 29; 39-46. DOI: https://doi.org/10.1016/j.ejon.2017.05.001

20. Enskär, K. Being an expert nurse in pediatric oncology care: nurses' descriptions in narratives. J. Pediatr. Oncol. Nurs. [Internet]. 2012 [cited 2018 Ago 5]; 29(3):151-60. DOI: https://doi.org/10.1177/1043454212446344

21. Toruner EK, Altay N. New Trends and Recent Care Approaches in Pediatric Oncology Nursing Asia-Pac. J. Oncol. Nurs. [Internet]. 2018 [cited 2018 Jun 13]; 5(2):156. DOI: https://doi.org/10.4103/apjon.apjon_3_18 